\title{
Wollen, Können und Dürfen der Kunden - Digitale Souveränität durch Kundenentwicklung
}

\author{
Denise Joecks-Laß ${ }^{(凶)}$ und Karsten Hadwich \\ Universität Hohenheim, Wollgrasweg 23, 70599 Stuttgart, Deutschland \\ denise.joecks-lass@uni-hohenheim.de
}

\begin{abstract}
Zusammenfassung. Der Einsatz von Informationstechnologien im Unternehmenskontext hat eine weitreichende Vernetzung von Unternehmen, Personen und Objekten zur Folge und erfordert entsprechend den Aufbau notwendiger technologischer Fähigkeiten und Ressourcen, um unabhängig und eigenständig zu handeln, d. h. digital souverän zu sein. Aufgrund der engen Zusammenarbeit von Anbieter und Kunde im Industriegüterkontext müssen Industriegüteranbieter nicht nur die eigene digitale Souveränität, sondern auch die digitale Souveränität des Kunden entwickeln. Wesentliche Voraussetzung der kundenseitigen digitalen Souveränität ist die Existenz der notwendigen Kompetenzen sowie das Vorhandensein des erforderlichen Wissens, um souverän handeln zu können. Die Kompetenzen und das Wissen können dabei von den Kunden zum einen selbst erlernt werden, zum anderen können Industriegüteranbieter durch eine systematische Kundenentwicklung den Aufbau der digitalen Souveränität von Kunden aktiv unterstützen. Ziel ist es daher, mögliche Instrumente der Kundenentwicklung zu analysieren, um den Kunden beim Aufbau von notwendigem Wissen und Kompetenzen für die Ausübung der digitalen Souveränität zu unterstützen.
\end{abstract}

Schlüsselwörter: Digitale Souveränität · Datensouveränität · Co-produktion · Kundenentwicklung · Customer Education

\section{Einleitung}

Neben der Globalisierung hat die Digitalisierung die Spielregeln in den letzten drei Jahrzehnten grundlegend verändert. Viele bestehende Wertschöpfungsketten und Geschäftsmodelle haben sich in der Folge entweder stark verändert oder sind gar weggefallen - zugleich sind neue entstanden (Bruhn/Hadwich 2017). Die damit verbundene Durchdringung von Informationstechnologien im persönlichen und beruflichen Alltag fördert dabei eine starke Vernetzung von Unternehmen, Personen und Objekten (Bruhn/Hadwich 2018), die zur Entwicklung von sogenannten Smart Products und Smart Services führen. Dabei handelt es sich um Produkte und Dienstleistungen, die mittels eines intelligenten Objektes Daten eigenständig austauschen 
(Allmendinger/Lombreglia 2005). Dies hat zur Folge, dass persönliche und unternehmensrelevante Daten mit Dritten geteilt werden (Axquisti et al. 2015). Entsprechend sind in den Unternehmen technologische Fähigkeiten und Ressourcen aufzubauen und Voraussetzungen für den Einsatz von neuen Technologien, den Zugang zu Kunden und den Austausch von Daten zu schaffen (Bogenstahl/Zinke 2017). Diese sogenannte digitale Souveränität bezieht sich dabei nicht nur auf den einzelnen Menschen, sondern auch auf gewerbliche, private und öffentliche Institutionen und Unternehmen (Bitkom 2015).

Der zunehmende Fokus - getrieben durch die Digitalisierung - auf der gemeinsamen Wertgenerierung von Anbieter und Kunde (Bruhn/Hadwich 2018) erfordert von Industriegüterunternehmen nicht nur die eigene digitale Souveränität, sondern auch die digitale Souveränität des Kunden zu entwickeln und damit die Voraussetzung für die Akzeptanz und Nutzung von technologiegetriebenen Dienstleistungen beim Kunden zu schaffen. Wesentliche Voraussetzung der kundenseitigen digitalen Souveränität ist die Existenz der notwendigen Kompetenzen sowie das Vorhandensein des erforderlichen Wissens, um souverän handeln zu können (Friedrichsen/Bisa 2016). Die Kompetenzen und das Wissen können von den Kunden zum einen selbst erlernt werden, zum anderen können Industriegüteranbieter durch eine systematische Kundenentwicklung den Aufbau der digitalen Souveränität bei Dienstleistungen von Kunden aktiv unterstützen. Dabei haben die Digitalisierung sowie die steigende Komplexität von digitalen Dienstleistungen den Bedarf an Kundenentwicklungsmaßnahmen in den letzten Jahren stetig steigen lassen (Youssef et al. 2018). Vor diesem Hintergrund diskutieren die folgenden Ausführungen die Sicherstellung der kundenseitigen digitalen Souveränität durch den Anbieter. Der Schwerpunkt liegt dabei auf den möglichen Instrumenten der Kundenentwicklung, um den Kunden beim Aufbau von notwendigem Wissen und Kompetenzen für die Ausübung der digitalen Souveränität zu unterstützen.

\section{Digitale Souveränität von Kunden}

\subsection{Digitale Souveränität - Eine Begriffsbestimmung}

Die Diskussion um die digitale Souveränität wurde in den vergangenen Jahren in der Praxis und Politik stetig vorangetrieben, befindet sich jedoch aus Forschungsperspektive in ihren Anfängen. So existiert kein einheitliches Begriffsverständnis (Friedrichsen/Bisa 2016; BITKOM 2015). Souveränität als Möglichkeit des selbstbestimmten Handelns zeichnet sich vor allem durch Eigenständigkeit und Unabhängigkeit aus (BITKOM 2015). Dabei wird digitale Souveränität zum Beispiel als ,das Bestimmungsrecht über alle persönlich digital erfassten Daten bzw. die eines Unternehmens“ (Friedrichsen/Bisa 2016, S. 41) definiert oder bezieht sich auf die Möglichkeit, Technologien selbstbestimmt zu nutzen (Bizer 2019). Insgesamt wird unter digitaler Souveränität das Bestimmungsrecht über die eigenen Daten (Friedrichsen/Bisa 2016) und die Selbstbestimmung bei der Technologienutzung verstanden. In der Folge werden neben dem Begriff der digitalen Souveränität die digitale Selbstbestimmung auch unter den Begriffen Datensouveränität und 
technologische Souveränität diskutiert (Couture/Toupin 2019). Beide stellen Teildimensionen der digitalen Souveränität dar, die sich auf konkrete Aspekte der Digitalisierung, z. B. den Austausch von Daten, beziehen. Zielsetzung dabei ist stets, die Befähigung zum selbstbestimmten Handeln in der digitalen Welt, entweder bei der Herstellung und der Nutzung von Technologien (technologische Souveränität) oder bei der Bereitstellung und der Nutzung von Daten (Datensouveränität).

Bisher ist bekannt, dass die Akzeptanz und Nutzungsintention von Technologien positiv beeinflusst werden, wenn die eingesetzte Technologie die Selbstbestimmung des Anwenders gewährleistet (Dupuy et al. 2016). Dabei konstituiert sich die Selbstbestimmung durch die Wahrnehmung der eigenen Autonomie und Kompetenzen sowie dem Gefühl von Zugehörigkeit (Deci/Ryan 2000). Daneben ist von Bedeutung, dass der Mensch sich als Quelle des eigenen Handelns versteht (Deci/Ryan 2000) und dass seine Kompetenzen ihn in die Lage versetzen, ein gewünschtes Verhalten durchzuführen bzw. zu kontrollieren (Bandura 1993). Selbstbestimmung ergibt sich demnach aus der Kombination von Kompetenzen, Fähigkeiten und Wissen, die einem ein Gefühl der Kontrolle und Einfluss auf die Situation ermöglichen. Digitale Souveränität bezeichnet demnach die wahrgenommene Fähigkeit, digitale Interaktions- und Transaktionsergebnisse zielgerichtet $\mathrm{zu}$ beeinflussen und $\mathrm{zu}$ kontrollieren, und befähigt dazu, selbstbestimmt und unabhängig zu handeln. Voraussetzungen stellen dabei Kompetenzen, Wissen und Fähigkeiten hinsichtlich der Datenverarbeitung und Technologienutzung dar.

\subsection{Digitale Souveränität als gemeinsame Co-Produktion}

Die enge Verflechtung eines Anbieters mit seinen Kunden im Industriegütersektor hat zur Folge, dass die anbieterseitige digitale Souveränität von dem Grad der digitalen Souveränität seiner Kunden abhängt. Ein Kunde, der sein Netzwerk sowie Daten nur unzureichend schützt, kann unbefugten Dritten unwissentlich einen Zugang zu Informationen des Anbieters gewähren. Daher sind Anbieter gezwungen, neben der eigenen digitalen Souveränität auch die digitale Souveränität ihrer Kunden zu betrachten. Dabei ist zu prüfen, in welchem Umfang Anbieter proaktiv bei der Entwicklung der kundenseitigen digitalen Souveränität unterstützen können.

Bereits Ende der 1970er-Jahre stellen Lovelock und Young (1979) heraus, dass Kunden eine produktive Ressource bzw. aktive Teilnehmer von Produktions- und Erstellungsprozessen sind. Der Kunde wird in der Folge Co-Produzent der Leistung und damit ein erfolgskritischer Potenzialfaktor (Bruhn et al. 2019). Ergreift ein Anbieter Maßnahmen, um die digitale Souveränität seiner Kunden erfolgreich zu entwickeln, stellt dies einen Entwicklungsprozess mit aktiver Kundenintegration dar. Erst die Zusammenarbeit zwischen Kunden und Anbieter führt zu der gewünschten nutzenstiftenden Wirkung (Grönroos/Voima 2013; Vargo/Lusch 2004). Aus Anbietersicht lassen sich Kosten- und Effizienzvorteile der Digitalisierung erst realisieren, wenn auch ihre Kunden ein ausreichendes Maß an digitalem Know-how besitzen. Hierfür sind zum einen (1) die Rollen und Aufgaben der Kunden und zum anderen (2) die notwendigen Kompetenzen und das Wissen $\mathrm{zu}$ definieren und zu vermitteln. Des Weiteren ist sicherzustellen, dass die Kunden (3) eine ausreichende Motivation besitzen, gemeinsam mit dem Anbieter zu kooperieren (Meuter et al. 2005). 
Der erste Schritt erfolgreicher Kundenintegration ist die Definition von Rollen und Aufgaben der Kunden (Bowers et al. 1990): Kunden benötigen einen Orientierungsrahmen, um zu wissen, was sie erwarten können und was sie dürfen (Meuter et al. 2005). Hierzu gehört die Definition der Aufgaben, Tätigkeiten und Entscheidungen, die der Kunde übernimmt (Bowers et al. 1990) und seiner digitalen Souveränität dienen. Um digital souverän zu handeln, benötigen Kunden Mitsprache- und Kontrollmöglichkeiten z. B. über Bedienung und Nutzung von Technologien oder der Verwendung von Daten.

Kompetenzen und Wissen bilden die wesentliche Voraussetzung für die Entwicklung von digitaler Souveränität (Friedrichsen/Bisa 2016) und erlauben dem Kunden, digitale Dienstleistungen und die zugrunde-liegenden Technologien zu verstehen und erfolgreich zu nutzen (Ganz et al. 2018). Zentrale Kompetenzen dabei sind z. B. solche, die im Zusammenhang mit der Datensammlung und-auswertung (Data Analytics) oder den Vernetzungsfähigkeiten von Menschen und Objekten (Lenka et al. 2017; Ritter/Pedersen 2020) stehen. Ebenfalls sind Wissen und Kompetenzen im Hinblick auf Gesetzgebung und Verträge (Ritter/Pedersen 2020) von Bedeutung. So sind z. B. im grenzübergreifenden Datenverkehr geografische Besonderheiten in der Gesetzgebung und Vertragsgestaltung $\mathrm{zu}$ berücksichtigen (BMWI 2017). Des Weiteren sind Kenntnisse im Zusammenhang mit der Interoperabilität von Technologien und deren Komponenten zu beachten (BMWI 2017). Zudem werden anspruchsvolle IT-Kenntnisse und Fähigkeiten zum kontinuierlichen Umgang mit Maschinen und Netzwerksystemen sowie deren Bedienung, Pflege und Instandhaltung benötigt (BMWI 2017; Ganz et al. 2018). Die Zusammenarbeit zwischen Anbieter und Kunde erfordert dabei ein ausreichendes Maß an Kommunikations-, Kooperations- und Koordinationsfähigkeiten (Ganz et al. 2013).

Grundsätzlich ist es die Entscheidung des Kunden, wie digital souverän dieser handelt und sein will. Das Verhalten und die Entscheidungen von Kunden hängen wesentlich von deren Motivation ab, gemeinsam mit dem Anbieter zu arbeiten (Meuter et al. 2005). Dabei ist zu unterscheiden, ob die Motivation, digital souverän zu handeln, aus eigenem inneren Wunsch (intrinsisch motiviert) oder durch externe Einflüsse (extrinsisch motiviert), z. B. durch finanzielle Anreize, erfolgt. Anbieter können dabei durch eine proaktive Kundenentwicklung die digitale Souveränität sicherstellen, indem sie die Rollen und Aufgaben definieren, notwendige Kompetenzen vermitteln sowie die Motivation zur Zusammenarbeit steigern.

\section{Entwicklung der kundenseitigen digitalen Souveränität}

\subsection{Ziele der Kundenentwicklung}

Ziel der Kundenentwicklung ist es, dem Kunden ausreichend Informationen und Wissen über Produkte und Dienstleistungen zu vermitteln, um das kundenseitige Verständnis zu steigern (Brunetti et al. 2016) und eine erfolgreiche Co-Produktion zwischen Anbieter und Kunden zu gewährleisten (Bitner et al. 1997; Hibbert et al. 2012; Bell et al. 2017). Kundenentwicklungsmaßnahmen sind Dienstleistungsangebote des Anbieters, die darauf abzielen, den Kunden ausreichend zu befähigen, 
damit dieser erfolgreich an Markttransaktionen teilnehmen kann (Brunetti et al. 2016). So können durch die Maßnahmen der Kundenentwicklung z. B. notwendiges Wissen vermittelt, Anforderungen an den Kunden kommuniziert und die Motivation zur Mitarbeit gesteigert werden (z. B. Huang et al. 2013; Hibbert et al. 2012).

Die proaktive Entwicklung und Schulung von Kunden wirkt sich dabei nachweislich positiv auf die Kundenzufriedenheit (z. B. Bell et al. 2017) und die Kundenbindung aus (z. B. Suh et al. 2015; Eisingerich/Bell 2006). Vor dem Hintergrund der besonderen Relevanz von Vertrauen im Kontext der Digitalisierung (Paeffgen/Eichen 2020) stellt die Kundenentwicklung eine Dienstleistung dar, die den Kunden bei der Digitalisierung unterstützt und die Kundenbeziehung weiter festigt (Suh et al. 2015). Zudem fördert die Kundenentwicklung die Akzeptanz sowie Nutzung von technologiegetriebenen Produkten und Dienstleistungen. Dabei wird das Nutzungsverhalten maßgeblich von den Fähigkeiten und Kompetenzen des Anwenders bestimmt (Aubert et al. 2005; Xue et al. 2007). Die eigenen Kunden beim Aufbau dieser Kompetenzen $\mathrm{zu}$ unterstützen, stellt vor diesem Hintergrund nicht nur eine Chance dar, die Geschäftsbeziehung weiter zu festigen, sondern aktiv die Diffusion und Akzeptanz der eigenen Dienstleistungen und den verbundenen Technologien voranzutreiben.

\subsection{Instrumente der Kundenentwicklung}

Kundenentwicklungsmaßnahmen stellen zum einen relevante Informationen zur Verfügung, z. B. im Hinblick auf die eingesetzten Technologien und Netzwerke, und schaffen zum anderen eine angemessene Lernumgebung für Kunden (Brunetti et al. 2016). Maßnahmen zur Kundenentwicklung beinhalten sowohl die Bereitstellung als auch die proaktive Vermittlung von Informationen. Dabei ist individuell festzulegen, welche Entwicklungsmaßnahmen für den jeweiligen Kunden geeignet sind (Burton 2002; Brunetti et al. 2016). So können Trainings durchgeführt oder Material zum Selbststudium bereitgestellt werden (Burton 2002).

Die unter dem Stichwort der Customer Education diskutierten Maßnahmen reichen dabei von professionellen Trainings, über Beratungen, bis hin zu Blogs, Seminaren, Foren, Werbung und anderen Offline- und Onlineaktivitäten (Suh et al. 2015). Grundsätzlich ist dabei zu unterscheiden, ob die Kundenentwicklung informations- oder erfahrungsbasiert erfolgt. Eine informationsbasierte Kundenentwicklung legt ihren Fokus auf die Vermittlung von Informationen, die der Kunde dann anwenden kann, während erfahrungsbasierte Entwicklungsansätze sich meist durch eine aktive Teilnahme und Ausprobieren des Kunden auszeichnen (Webb 2000; Gouthier 2003). Analog zur Entwicklung von Mitarbeitern (Becker 2010), werden die Instrumente der Kundenentwicklung eingesetzt, um die Motivation (Wollen) oder die Qualifizierung von Kunden (Wissen und Können) zu fördern. Zudem stellt die Gestaltung des Handlungsspielraums und der Lernumgebung (Dürfen) ein weiteres Handlungsfeld der Kundenentwicklung dar (Gouthier 2003).

\subsection{Digitale Souveränität durch Kundenentwicklung}

Digitale Souveränität erfordert die gemeinsame Co-Produktion von Anbieter und Kunden. Folglich sind Aufgaben und Kompetenzprofile zu erstellen und die 
Motivation zur Zusammenarbeit sicherzustellen. Jeder dieser Faktoren lässt sich einer bestimmten Stellschraube der Kundenentwicklung zuordnen (vgl. Abb. 1). Neben den Maßnahmen, die auf das Wollen (Motivation der Kunden), das Wissen und Können (Kompetenzen der digitalen Souveränität) sowie das Dürfen (Tätigkeiten, Aufgaben und Entscheidungsspielraum der Kunden) abzielen, ist für eine erfolgreiche Entwicklung der kundenseitigen digitalen Souveränität und die Ausgestaltung einer angenehmen Lernumgebung von Bedeutung (Gouthier 2003). Die Ausgestaltung der Kundenkontaktpunkte im Rahmen der Qualifizierungsmaßnahmen beeinflusst dabei die Lernbereitschaft und -fähigkeit der Kunden sowohl positiv als auch negativ (Fassot 1995).

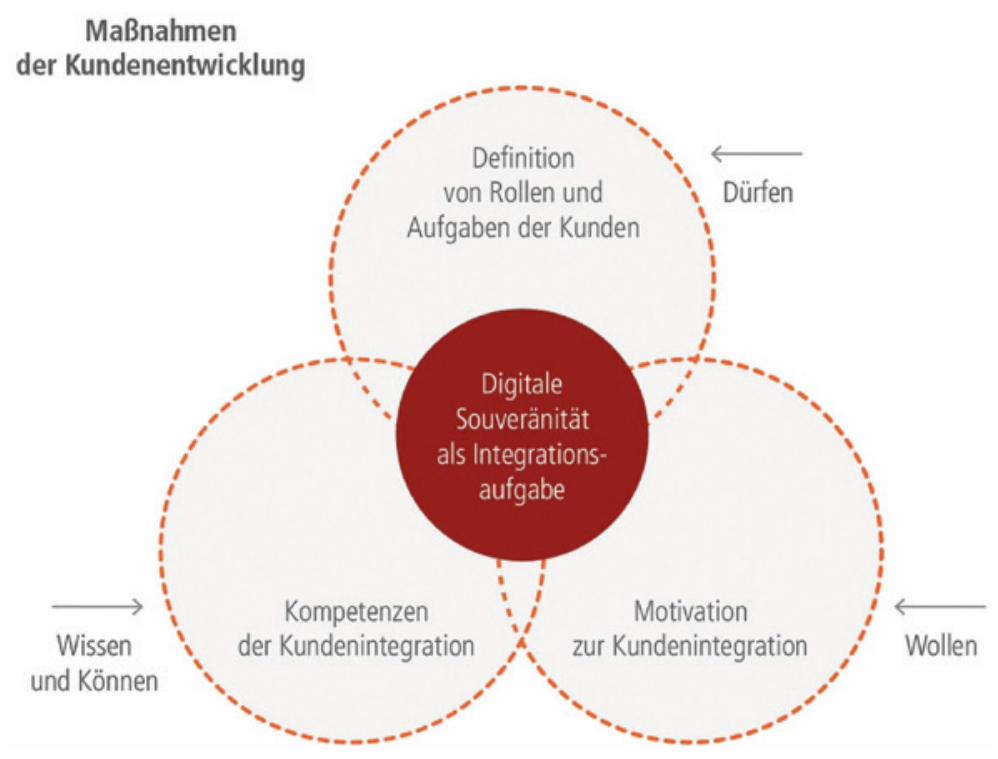

Abb. 1. Ausgestaltung der Kundenentwicklung zur Sicherstellung der digitalen Souveränität

\section{Definition von Rollen und Aufgaben der Kunden (Dürfen der digitalen Souveränität)}

Digitale Souveränität als gemeinsame Integrationsaufgabe von Anbieter und Kunde erfordert die Definition von Aufgaben, Rollen und Erwartungen (Meuter et al. 2005). Dabei werden unter dem Stichwort „Dürfen“ in der Kundenentwicklung die Maßnahmen verstanden, die den Handlungsspielraum der Kunden definieren (Gouthier 2003). Ein für die Entwicklung der kundenseitigen digitalen Souveränität effektives Instrument ist die Definition der kundenseitigen Tätigkeiten sowie die Festlegung der Entscheidungs- und Kontrollspielräume (Oechsler 2015). Erstere beziehen sich auf den Umfang der von Kunden erbrachten Leistungen während Letztere das Maß des autonomen Handelns bestimmen (Gouthier 2003). So können Aufgaben, die die digitale Souveränität des Kunden einschränken, von diesem direkt übernommen 
werden, wie z. B. die Analyse und die Auswertung von Daten. Um digital selbstbestimmt zu handeln, ist der Grad des autonomen Handelns ebenfalls von Bedeutung. Für die digitale Souveränität ist ein ausreichendes Maß an Mitbestimmung z. B. über die Weitergabe von Daten und Informationen bis hin zur gemeinsamen Technologieentscheidung notwendig. Selbstbestimmung erfordert die aktive Teilnahme an Entscheidungsprozessen, um deren Folgen richtig einschätzen zu können. Die Entscheidungen über die Tätigkeiten, Aufgaben sowie den Grad des autonomen Handelns unterstützen dabei aktiv die spezifische Rollen- und Aufgabendefinition der kundenseitigen digitalen Souveränität.

\section{Kompetenzen der Kundenintegration (Wissen und Können der digitalen Souveränität)}

Grundsätzlich ist bei der Umsetzung der Kundenentwicklung der digitalen Souveränität zu unterscheiden, ob die Maßnahmen die Wissensvermittlung (Wissen) oder den Aufbau von Kompetenzen und Fertigkeiten (Können) verfolgen. Anbietern stehen dabei verschiedene Kommunikationsinstrumente, Trainings- und Lehrformate zur Verfügung (Burton 2002; Suh et al. 2005).

Um selbstbestimmt bei digitalen Transaktionen $\mathrm{zu}$ handeln, ist auf Seiten der Kunden Wissen notwendig, z. B. in Hinblick auf die Funktionsweise der eingesetzten Technologie sowie die rechtlichen Rahmenbedingungen. Wissen über die Datensammlung und -verarbeitung ist ebenfalls für die digitale Selbstbestimmung von Interesse. Um Wissen zu vermitteln, können dem Kunden Schulungs- und Trainingsangebote angeboten werden (Suh et al. 2015). Zudem spielt im Industriegüterkontext auch der individuelle persönliche Kontakt von Unternehmen und Kunden eine bedeutende Rolle (Lilien 2016), um relevantes Wissen, z. B. in Hinblick auf die Instandhaltung und Nutzung der technischen Systeme, zu vermitteln. Für die Wissensvermittlung sind auch Kommunikationsmaßnahmen aus dem Marketing oder Vertrieb relevant, wie z. B. eine Website, Broschüren und Vorträge (Burton 2002; Gouthier 2003; Suh et al. 2015).

Hinsichtlich der Vermittlung von Kompetenzen und Fertigkeiten sind vor allem Trainings (online und offline) von Bedeutung. Zudem können Schulungen oder Seminare angeboten werden, um die Kunden z. B. in Analysemethoden und deren Funktionsweisen einzuarbeiten, um so den Kunden Kontrolle und Entscheidungshoheit über ihre Daten zu ermöglichen. Ebenfalls von Bedeutung sind Beratungsgespräche durch den Anbieter (Gouthier 2003; Suh et al. 2015). In einer Eins-zu-eins-Situation können Kunden unterrichtet werden, z. B. im Umgang mit Verfahren und Methoden zur Vernetzung von Personen und Objekten über Unternehmensgrenzen hinweg.

\section{Motivation zur Kundenintegration (Wollen der digitalen Souveränität)}

Voraussetzung zur Entwicklung der digitalen Souveränität ist die Motivation des Kunden. Daher sind Maßnahmen zu ergreifen und Anreize zu identifizieren, die Kunden motivieren (Wollen), digital souverän zu handeln (Gouthier 2003). Aufgrund der wachsenden Skepsis und Bedenken hinsichtlich der Netzwerksicherheit und des Datenschutzes (Vakeel et al. 2017) ist anzunehmen, dass Kunden ein grundsätzliches Interesse an der Sicherstellung der eigenen digitalen Souveränität besitzen (intrinsische Motivation der digitalen Souveränität). Die mit der digitalen 
Souveränität einhergehenden Kompetenzen ermöglichen den Kunden, eine bessere Kontrolle über die eigenen Daten auszuüben und als gleichberechtigte Partner in Transaktionen mit unterschiedlichen Akteuren zu agieren. Zusätzlich können Anbieter zur Steigerung der Motivation Verhaltensanreize setzen, die entweder negativ (in Form von Bestrafungen) oder positiv (in Form von Belohnungen) ausgestaltet sind (Higgins 2000). In Bezug auf die digitale Souveränität sind vor allem Anreizmechanismen einzusetzen, die dem Kunden zum einen den Mehrwert der digitalen Souveränität aufzeigen und zum anderen finanzielle Vorteile bieten. Beispiel für solche Anreizinstrumente sind z. B. Rabatte oder kostenlose Nutzungsrechte. Nichtfinanzielle Anreize stellen im Kontext der digitalen Souveränität z. B. das Gefühl von Sicherheit dar. Vertrauensvolle Beziehungen zwischen Anbieter und Kunden helfen ebenfalls, den Kunden zu motivieren, seine digitale Souveränität sicherzustellen.

Zusammenfassend stehen den Anbietern eine Vielzahl an unterschiedlichen Instrumenten und Maßnahmen zur Verfügung, um ihren Kunden bei der Entwicklung der digitalen Souveränität zu unterstützen (vgl. Abb. 2). Neben Maßnahmen, die das Lernen von Inhalten und Fertigkeiten ermöglichen, spielen aber auch Kontaktsituationen zwischen Anbieter und Kunde sowie die Spezifikation des kundenseitigen Handlungsspielraums eine wesentliche Rolle bei der Entwicklung der kundenseitigen digitalen Souveränität durch den Anbieter.

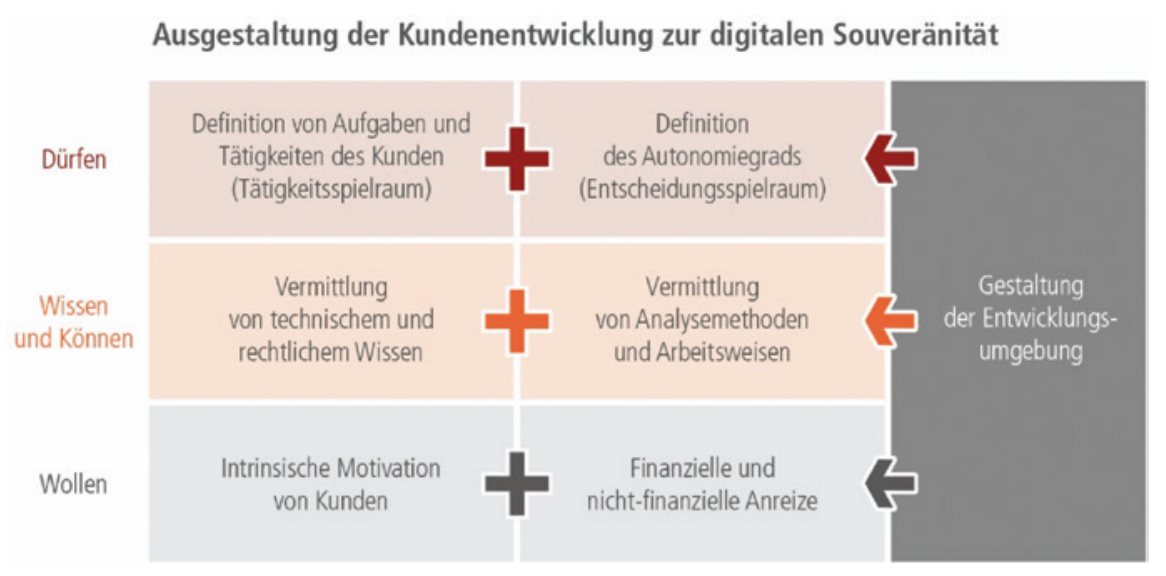

Abb. 2. Ausgestaltung der Kundenentwicklung zur digitalen Souveränität

\section{Fazit und Ausblick}

Die Digitalisierung erfordert ständiges Lernen und die Entwicklung neuer Kompetenzen. Eine wesentliche Herausforderung ist dabei die Sicherstellung der digitalen Selbstbestimmung bzw. digitalen Souveränität sowohl für Unternehmen als auch deren Kunden. Dabei besitzen die Anbieter die Möglichkeit, ihre Kunden aktiv bei der Entwicklung der digitalen Souveränität durch geeignete 
Kundenentwicklungsmaßnahmen zu unterstützen, indem sie die Motivation der Kunden (Wollen) aktiv fördern und dem Kunden relevante Qualifikationen (Wissen und Können) vermitteln. Zudem werden Anbieter durch die Bereitstellung passender Lernumgebungen sowie die Erweiterung des Handlungs- und Tätigkeitsraums des Kunden (Dürfen) in die Lage versetzt, die digitale Souveränität von Kunden auszubauen. In diesem Kontext ist vor allem von Bedeutung, den Kunden mit dem notwendigen Wissen in Hinblick auf die Funktionsweise und Nutzung unterschiedlicher Technologien auszustatten sowie ausreichende Methodenkompetenzen in Hinblick auf Datenanalyse und Methoden zur Vernetzung von Personen und Objekten zu vermitteln.

Die rasch fortschreitende Digitalisierung wird dabei den Wunsch, die eigene digitale Souveränität sicherzustellen, wachsen lassen. Dabei wird es zukünftig von Bedeutung sein, den Grad der digitalen Souveränität bestimmen zu können, um ausgehend davon, die erforderlichen Maßnahmen zum Ausbau der digitalen Souveränität zu identifizieren und zu planen - sowohl aus Sicht des Anbieters als auch des Kunden. In diesem Kontext stellt sich zudem die Frage, welche Interdependenzen zwischen den unterschiedlichen Graden der digitalen Souveränität von verschiedenen Akteuren existieren. Zudem ergeben sich Herausforderungen bei der Sicherstellung der digitalen Souveränität im Kontext von externen Unternehmensnetzwerken. Ein weiteres relevantes Entwicklungsfeld ist Datensouveränität. Daten als Motor von neuen digitalen Geschäftsmodellen und wesentlicher Treiber des Unternehmenserfolgs stehen den wachsenden Bedenken hinsichtlich der Datenverarbeitung auf Kundenseite (Gimpel et al. 2018) sowie den Bestrebungen von Regierungen, die Verarbeitung von Daten zu regulieren (z. B. durch die Datenschutzgrundverordnung) gegenüber. Hier wird es zukünftig von Interesse sein, diejenigen Maßnahmen und Faktoren zu identifizieren, die die Datensouveränität sowie deren Wahrnehmung maßgeblich verbessern.

Schlussendlich werden der wachsende Datenverkehr, die Entwicklung neuer digitaler Geschäftsmodelle und der weitreichende Einsatz von Informationstechnologien das wirtschaftliche und gesellschaftliche Leben weiter verändern und vorantreiben. Ein proaktiver Umgang mit den damit verbundenen Herausforderungen sowie die Entwicklung und Sicherstellung der digitalen Souveränität werden somit zukünftig wesentliche Erfolgsgrößen darstellen.

\section{Literatur}

Allmendinger, G., Lombreglia, R.: Four strategies for the age of smart services. Harv. Bus. Rev. 83(10), 131-145 (2005)

Aubert, B, Khoury, G., Jaber, R.: Enhancing customer relationships through customer education: an exploratory study. In: Proceedings of the 1st International Conference on E-Business and E-Learning, S. 194-201. Athen (2005)

Axquisti, A., Brandimarte, L., Loewenstein, G.: Privacy and human behavior in the age of information. Science 347(6221), 509-514 (2015)

Bandura, A.: Perceived self-efficacy in cognitive development and functioning. Educ. Psychol. 28(2), 117-148 (1993) 
Becker, M.: Entwicklungstendenzen der Personalentwicklung Personalentwicklung 2015. In: Wagner, D., Herlt, S. (Hrsg.) Perspektiven des Personalmanagement 2015, S. 233-266. Springer Gabler, Wiesbaden (2010)

Bowers, M.R., Martin, C.L., Luker, A.: Trading places: employees as customers, customers as employees. J. Serv. Mark. 4(2), 55-69 (1990)

Bell, S.J., Auh, S., Eisingerich, A.B.: Unraveling the customer education paradox. J. Serv. Res. 20(3), 306-321 (2017)

BITKOM: Digitale Souveränität - Positionsbestimmung und erste Handlungsempfehlungen für Deutschland und Europa (2015). https://doi.org/10.1007/s11623-018-0944-y

Bitner, M.J., Franda, W.T., Hubbert, A.R., Zeithaml, V.A.: Customer contribution and roles in service delivery. Int. J. Serv. Ind. Manag. 8(3), 193-205 (1997)

Bizer, J.: Digitale Souveränität: Wer steuert, organisiert und kontrolliert die digitale Verwaltung? In: Lühr, H.H., Jabkowski, R., Smentek, S. (Hrsg.) Handbuch Digitale Verwaltung, S. 23-26. KSV Verwaltungspraxis, Wiesbaden (2019)

Bogenstahl, C., Zinke, G.: Digitale Souveränität: Ein mehrdimensionales Handlungskonzept für die deutsche Wirtschaft. In: Wittpahl, V. (Hrsg.) Digitale Souveränität: Bürger, Unternehmen, Staat, S. 65-82. Springer, Wiesbaden (2017)

BMWI: Kompetenzen für eine Digitale Souveränität (2017). https://www.bmwi.de/Redaktion/ DE/Publikationen/Studien/kompetenzen-fuer-eine-digitale-souveraenitaet.html. Zugegriffen: 12. Juni 2017

Bruhn, M., Hadwich, K.: Servicetransformation - Eine Einführung in die theoretischen und praktischen Problemstellungen. In: Bruhn, M., Hadwich, K. (Hrsg.) Servicetransformation - Entwicklung vom Produktanbieter zum Dienstleistungsmanagement, S. 3-22. Springer, Wiesbaden (2017)

Bruhn, M., Hadwich, K.: Dienstleistungen 4.0 - Erscheinungsformen, Transformationsprozesse und Managementimplikationen. In: Bruhn, M., Hadwich, K. (Hrsg.) Dienstleistungen 4.0: Geschäftsmodelle - Wertschöpfung - Transformation, S. 1-39. Springer, Wiesbaden (2018)

Bruhn, M., Meffert, H., Hadwich, K.: Handbuch Dienstleistungsmarketing: Umsetzung Planung - Kontrolle, 2. Aufl. Springer, Wiesbaden (2019)

Brunetti, F., Bonfanti, A., Vigolo, V.: Empowering customer education: a research agenda for marketing studies. In: 9th Annual Conference of the EuroMed Academy of Business, S. 393-405. EuroMed, Warschau (2016)

Burton, D.: Consumer education and service quality: conceptual issues and practical impliations. J. Serv. Mark. 16(2), 125-142 (2002)

Couture, S., Toupin, S.: What does the notion of 'Sovereignty' mean when referring to the digital? New Media Soc. 21(10), 2305-2322 (2019)

Deci, E., Ryan, R.M.: The "What" and "Why" of goal pursuits: human needs and the selfdetermination of behavior. Psychol. Inq. 11(4), 227-268 (2000)

Dupuy, L., Consel, C., Sauzéon, H.: Self-determination-based design to achieve acceptance of assisted living technologies for older adults. Comput. Hum. Behav. 65, 508-521 (2016)

Eisingerich, A.B., Bell, S.J.: Relationship marketing in the financial services industry: the importance of customer education, participation and problem management for customer loyalty. J. Finan. Serv. Mark. 10(4), 86-97 (2006)

Fassot, G.: Dienstleistungspolitik industrieller Unternehmen. Springer, Wiesbaden (1995) 
Friedrichsen, M., Bisa, P.: Einführung - Analyse der digitalen Souveränität auf fünf Ebenen. In: Friedrichsen, M., Bisa, P.J. (Hrsg.) Digitale Souveränität: Vertrauen in der Netzwerkgesellschaft. Springer Fachmedien, Wiesbaden (2016)

Ganz, W., Dworschak, B., Schnalzer, K.: Competences and competences development in a digitalized world of work. In: Nunes, I.L. (Hrsg.) AHFE: International Conference on Applied Human Factors and Ergonomics - Advances in Human Factors and System Interaction, S. 312-320. Springer, Los Angeles (2018)

Ganz, W., Tombeil, A.S., Bornewasser, M., Theis, P.: Produktivität von Dienstleistungsarbeit. Frauenhofer, Stuttgart (2013)

Gimpel, H., Kleindienst, D., Nüske, N., Rau, D., Schmied, F.: The upside of data privacy delighting customers by implementing data privacy measures. Electron. Mark. 28(4), 437452 (2018)

Gouthier, M.H.J.: Kundenentwicklung im Dienstleistungsbereich. Deutscher Universitäts, Wiesbaden (2003)

Grönroos, C., Voima, P.: Critical service logic: making sense of value creation and co-creation. J. Acad. Mark. Sci. 41(2), 133-150 (2013)

Hibbert, S., Winklhofer, H., Temerak, M.S.: Customers as resource integrators. J. Serv. Res. 15(3), 247-261 (2012)

Higgins, E.T.: Making a good decision: value from fit. Am. Psychol. 55, 1217-1230 (2000)

Huang, M.-X., Huang, Y., Deng, Y.-N.: How to improve customer participation through customer education: from the perspective of customer readiness. In: 6th International Conference on Information Management, Innovation Management and Industrial Engineering, S. 251-254. Xi' an (2013)

Lenka, S., Parida, V., Wincent, J.: Digitalization capabilities as enablers of value co-creation in servitizing firms. Psychol. Mark. 34(1), 92-100 (2017)

Lilien, G.L.: The B2B knowledge gap. Int. J. Res. Mark. 33(3), 543-556 (2016)

Lovelock, C.H., Young, R.F.: Look to consumers to increase productivity (1979). https://hbr. org/1979/05/look-to-consumers-to-increase-productivity

Meuter, M.L., Bitner, M.J., Ostrom, A.L., Brown, S.W.: Choosing among alternative service delivery modes: an investigation of customer trial of self-service technologies. J. Mark. 69(April), 61-83 (2005)

Oechsler, W.A.: Personal und Arbeit, 10. Aufl. DeGryuter, Berlin (2015)

Paeffgen, N., Eichen, F.: Digital trust from the customer's perspective: a qualitative study in Switzerland (2020). https://digitalswitzerland.com/wp-content/uploads/2020/01/BookletsKey-Findings-Digital-Trust.pdf

Ritter, T., Pedersen, C.L.: Digitization capability and the digitization of business models in business-to-business firms: past, present, and future. Ind. Mark. Manage. 86, 180-190 (2020)

Suh, M., Greene, H., Israilov, B., Rho, T.: The impact of customer education on customer loyalty through service quality. Serv. Mark. Q. 36, 261-280 (2015)

Vakeel, K.A., Das, S., Udo, G.J., Bagchi, K.: Do security and privacy policies in B2B and B2C E-Commerce differ? A comparative study using content analysis. Behav. Inform. Technol. 36(4), 390-403 (2017)

Webb, D.: Understanding customer roles and its importance in the formation of service quality expectations. Serv. Ind. J. 20(1), 1-12 (2000) 
Vargo, S., Lusch, R.F.: Evolving to a new dominant logic for marketing. J. Mark. 68(1), 1-17 (2004)

Xue, M., Hitt, L.M., Harker, P.T.: Customer efficiency, channel usage, and firm performance in retail banking. Manuf. Serv. Oper. Manag. 9(4), 535-558 (2007)

Youssef, K.B., Viassone, M., Kitchen, P.: Exploring the relationship between education and customer satisfaction. Ital. J. Manag. 36(105), 43-60 (2018)

Open Access Dieses Kapitel wird unter der Creative Commons Namensnennung 4.0 International Lizenz (http://creativecommons.org/licenses/by/4.0/deed.de) veröffentlicht, welche die Nutzung, Vervielfältigung, Bearbeitung, Verbreitung und Wiedergabe in jeglichem Medium und Format erlaubt, sofern Sie den/die ursprünglichen Autor(en) und die Quelle ordnungsgemäß nennen, einen Link zur Creative Commons Lizenz beifügen und angeben, ob Änderungen vorgenommen wurden.

Die in diesem Kapitel enthaltenen Bilder und sonstiges Drittmaterial unterliegen ebenfalls der genannten Creative Commons Lizenz, sofern sich aus der Abbildungslegende nichts anderes ergibt. Sofern das betreffende Material nicht unter der genannten Creative Commons Lizenz steht und die betreffende Handlung nicht nach gesetzlichen Vorschriften erlaubt ist, ist für die oben aufgeführten Weiterverwendungen des Materials die Einwilligung des jeweiligen Rechteinhabers einzuholen. 